\title{
RESONANT ABSORPTION OF P-MODES BY SUNSPOTS
}

\author{
S. M. CHITRE ${ }^{1}$ and JOSEPH M. DAVILA \\ Laboratory for Astronomy and Solar Physics \\ NASA-Goddard Space Flight Center \\ Greenbelt, MD 20771
}

\section{Introduction}

A substantial amount $(\lesssim 50 \%)$ of solar acoustic (p-mode) absorption in sunspot regions has been reported by Braun, Duvall and LaBonte (1988). A number of suggestions have been advanced to account for the observed p-mode absorption in sunspots (see review by Davila in these proceedings). These include processes involving resonance absorption, radiation damping, and non-dissipative mechanisms. The main purpose of the present work is to demonstrate that any dissipative process like radiative, viscous, or resistive dissipation leads to the resonant absorption of acoustic waves incident on the sunspot tube and that the resultant heating rate can be shown to be consistent with the observed absorption of the p-mode power impinging on an isolated inhomogeneously structured sunspot.

\section{Model Assumptions and Governing Equations}

Sunspot is assumed to be a localized cylindrical $(r, \theta, z)$ flux-tube bounded by $r=R_{\text {spot }}$, which is in transverse pressure equilibrium with a field-free homogeneous medium. The spot magnetic field is taken to be vertical ( $z$-direction) with a dependence on the radial coordinate, $r$, only, i.e. $\mathbf{B}_{\mathbf{0}}=\left(0,0, B_{0}(r)\right)$. The equilibrium physical quantities; pressure, $p_{0}$, density, $\rho_{0}$, and temperature, $T_{0}$, are assumed to be functions of $r$, but gravity and vertical stratification are neglected. No specific assumption is made about the magnitude of $\beta=8 \pi p / B^{2}$. For the sake of illustration, the fluid in the flux tube is taken to be fully compressible and inviscid, with infinite electrical conductivity but with finite thermal (radiative) conductivity. However the results discussed do not depend on the specific dissipation mechanism. The basic equations are the usual conservation equations which can be linearized and then combined to get a fourth-order differential equation for the perturbed variables.

The heating rate may be calculated by integrating the incident Poynting and acoustic flux over the surface of the resonance layer (Davila, 1987). Given the existence of the resonance layer in the spot tube (resulting from some dissipation process in the plasma) it suffices to compute the heating rate by invoking the ideal MHD solution that is valid on the surface and outside the resonance layer. The resultant heating rate per unit area contributed by the Poynting and acoustic flux comes out to be

$$
H=\frac{\rho_{0}}{2 \omega}\left[v_{A}^{2} \frac{k_{z}^{2} r^{2}}{m^{2}}\left(-\frac{1}{v_{A}^{2}} \frac{d v_{A}^{2}}{d r}\right)\right] \pi|V|^{2}
$$

All the physical quantities here are measured at the resonance layer. With a view to determine

\footnotetext{
${ }^{1}$ NAS-NRC Resident Research Associate on leave from Tata Institute of Fundamental Research, Bombay
} 
whether this heating rate is consistent with the observed absorption of $\mathrm{p}$-mode power, we equate $\mathrm{H}$ to $\alpha_{\max }=<\frac{1}{2} \rho_{0}^{\prime}\left(V_{\text {in }}^{2}-V_{\text {out }}^{2}\right) c_{s}>$, the maximum observed difference between the incoming and out-going acoustic flux. Here $V_{\text {in }}$ is the amplitude of the incoming p-wave, $V_{\text {out }}$ of the reflected wave, and $\rho_{0}^{\prime}$ is the density at the surface of the spot tube.

Choosing typical values for the sunspot tube; $c_{s}=10^{6} \mathrm{~cm}-\mathrm{s}^{-1}, v_{A}=3 \times 10^{6} \mathrm{~cm}-\mathrm{s}^{-1}$, $k_{z}^{2} r^{2} / \mathrm{m}^{2}=0.5, R=0.5, \nu=3.3 \mathrm{mHz},\left|\left(1 / v_{A}^{2}\right)\left(d v_{A}^{2} / d r\right) \quad\right|=1 / R_{s p o t}=2 \times 10^{-9} \mathrm{~cm}^{-1}$, and $\rho_{0}^{\prime} / \rho_{0}=2$, we get the wave amplitude,

$$
|V| \simeq \frac{1}{5} V_{\text {in }} \simeq 100 m-s^{-1}
$$

assuming a typical RMS value of $400 m-s^{-1}$ for the photospheric velocity amplitude of p-modes.

\section{Discussion and Conclusions}

The required heating rate yields the wave amplitude of the order of $100 m-s^{-1}$ at the resonance layer, which should be observable within the spot-tube at the surface. The heating rate scales like $1 / \mathrm{m}^{2}$ and this is also borne out by the detailed numerical calculation of the absorption coefficient by Lou (1989). Lou has examined the influence of viscous dissipation on the Alfven resonance; we expect that the absorption coefficient should be independent of the precise dissipation mechanism.

The solution to the problem of the absorption of axisymmetric modes may be sought by considering more realistic sunspot models. An isolated single spot is not expected to be a perfectly circular absorber or scatterer. The cylindrical waves converging onto a non-axisymmetric spot will inevitably couple the $m=0$ mode to $m>0$ modes. It is also unreasonable to imagine that a sunspot would remain as a vertical monolithic structure. The sunspot may be made up of a number of individual flux tubes which could also couple the modes. Finally, it can be demonstrated that the Alfven resonance, responsible for resonant heating, survives even when $\mathrm{m}=0$ when the flux tube is twisted (see also Hollweg, 1988).

\section{References}

Braun, D. C., T. L. Duvall, and B. J. LaBonte, 1988, Ap. J., 335, 1015.

Davila, J. M., 1987, Ap. J., 317, 514.

Hollweg, J. V., 1988, Ap. J., 335, 1005.

Lou, Y., 1989, Viscous Magnetohydrodynamic Modes and p-Mode Absorption by Sunspots, in press. 